\title{
Likely Residual Metabolic Tumor Activity
}

National Cancer Institute

\section{Source}

National Cancer Institute. Likely Residual Metabolic Tumor Activity. NCI Thesaurus. Code C123409.

A finding of focal metabolic activity at the primary or nodal sites that is greater than liver uptake. 